\title{
Erros de medicação realizados pelo técnico de enfermagem na UTI: contextualização da problemática
}

Recebido em: 20/04/2011

Aceito em: 15/02/2012

\author{
Bruna Correia Lopes ${ }^{1}$ \\ Mara Ambrosina de Oliveira Vargas ${ }^{2}$ \\ Nára Selaimen Gaertner Azeredo ${ }^{3}$ \\ Andressa Behenck ${ }^{4}$
}

Pesquisa qualitativa, exploratória, cujos objetivos foram descrever os tipos de erros ocorridos na administração de medicamentos pelos técnicos de enfermagem que trabalham em UTI geral e identificar a(s) justificativa(s) para a ocorrência de tais falhas. Após aprovação do Comitê de Ética e Pesquisa, a coleta de dados efetivou-se por entrevista semiestruturada com técnicos de enfermagem. Os resultados indicam que os erros mais relatados estão relacionados à preparação dos medicamentos, e as justificativas para a ocorrência de erros evidenciam a sobrecarga de trabalho e a falta de atenção, articuladas à inexperiência de alguns profissionais e às falhas na estrutura. Conclui-se que é premente otimizar estratégias preventivas para evitar ou minimizar erros com medicamentos.

Descritores: Enfermagem, Unidade de Terapia Intensiva, Segurança, Ética, Erros de Medicação.

\section{Medication errors made by the nursing technician in the ICU: contextualization of the problem}

That is an exploratory research with a qualitative focus, whose aim was to describe the types of errors that occur in the administration of medicines by the nursing technicians that work in the general Intensive Therapy Unit and identify the justification(s) for the occurences of such failures. After the approval of the Ethics and Research Committee of the institution hosting the study, the collected data was obtained by means of semi-structured interviews with nursing technicians. The results indicate that the most reported errors are related to the preparation of the medicines, and the justifications to the occurences of the errors highlight the work overload and the lack of attention, linked with the inexperience of some professionals and structure failures. It is concluded that is urgent the adoption of preventive strategies to avoid or to minimize errors with medicines.

Descriptors: Nursing, Intensive Therapy Unit, Safety, Ethics, Medicine Errors.

\section{Los errores de medicación realizados por el técnico de enfermería en la UTI: contextualización del problema}

Se trata de una investigación cualitativa, exploratória, cuyos objetivos fueron: describir los tipos de errores en la administración medicamentos por parte de personal de enfermería que trabajan en una unidad de cuidados intensivos general y para identificar a la persona (s) de la justificación (s) para la ocurrencia de dichos fallos. Tras la aprobación por el Comité de Ética e Investigación, recopilación de datos se realizó mediante entrevistas semi-estructuradas con el personal de enfermería. Los resultados indican que la mayoría de los errores reportados están relacionados con la preparación de medicamentos, y los motivos de los errores que ocurren destacan la sobrecarga de trabajo y la falta de atención, articulados a la inexperiencia de algunos profesionales y fallas en la estructura. Se concluye que existe una necesidad apremiante estrategias de optimización de prevención para evitar o reducir al mínimo los errores de la droga.

Descriptores: Enfermería, Unidad de Cuidados Intensivos, Seguridad, Ética, Errores de Medicación.

\section{INTRODUÇÃO}

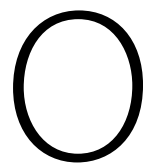
procedimentos de enfermagem, antes considerados "simples", como mudanças de decúbito, higiene oral, higiene corporal, cuidados com feridas, administração de medicamentos, hoje são avaliados como fundamentais para a recuperação do paciente. Ou seja, esses procedimentos exigem da enfermagem uma dimensão do cuidado até então não experimentada. Atualmente, considera-se que, se 0 cuidado é indevidamente realizado, aumentam o período de internação e o risco de adquirir infecção hospitalar e lesões que podem ser temporárias ou permanentes e, nos piores casos, evoluir para a morte. Acrescentam-se a esses acometimentos o prejuízo financeiro e os danos à própria equipe de enfermagem ${ }^{(1 ; 2)}$. Portanto, a segurança do paciente tem merecido crescente atenção dos enfermeiros, pela busca de qualidade e exposição mínima a riscos ${ }^{(3)}$.

1 Enfermeira com graduação em enfermagem pela Universidade do Vale do Rio dos Sinos (Unisinos). Atua no Hospital da PUC.

2 Enfermeira e doutora em enfermagem com ênfase em filosofia, saúde e sociedade pela Universidade Federal de Santa Catarina (UFSC). Professora adjunta de graduação e pós-graduação em enfermagem da UFSC. E-mail: maraav@terra.com.br

3 Enfermeira e mestre em educação e saúde pela Faculdade de Medicina da Universidade Federal do Rio Grande do Sul (UFRGS). Coordenadora de enfermagem da UTI do Hospital Nossa Senhora da Conceição (GHC/MS).

4 Enfermeira, atua no Hospital de Clínicas de Porto Alegre (HCPA). Graduação em enfermagem pelo IPA. 
Os erros de medicação estão entre os que representam um sério problema para os pacientes, para os profissionais de saúde e para a instituição. Ou seja, todos são prejudicados com esse tipo de falha ${ }^{(4)}$. Nessa perspectiva, o reflexo do erro atinge a instituição, comprometendo sua imagem na qualidade do atendimento, e provoca desconfiança nos clientes - que, quando necessário, não retornam à instituição -, além de gerar custos elevados, multas e processos. Para os profissionais da saúde, há punições que vão desde advertências verbais e/ou escritas, demissões e algumas vezes enfrentamento de processos civis, legais e éticos. O resultado disso, em algumas situações, é o impedimento do exercício legal da profissão e custos emocionais, como sentimento de culpa, depreciação da imagem e demissão voluntária(2)

Por diversas vezes, o erro de medicação só é detectado após a administração da droga, quando o paciente começa a apresentar alterações não esperadas para seu quadro clínico, alertando a equipe e o responsável pelo paciente naquele momento. Concomitante $a$ isso, a cultura de punir o profissional prepondera, dificultando, assim, que ele exponha o erro e que medidas preventivas sejam implantadas. Por causa disso, somente $25 \%$ dos erros são relatados à instituição. O profissional da saúde vivencia o medo de punições, demissão, processos civis, penais e éticos e, claro, a preocupação com a gravidade do erro e a vergonha ${ }^{(5)}$.

A justificativa da escolha desse tema acontece por se tratar de uma preocupação mobilizada por parte dos profissionais de saúde em geral, já que a questão da segurança, das práticas seguras e da biossegurança é uma demanda atualizada. Os hospitais tornaram-se, por diferentes motivos, áreas de risco. Preconiza-se como máxima que a busca da segurança do paciente implica ou impacta na qualidade do cuidado de enfermagem ${ }^{(1)}$. Existem poucos trabalhos publicados sobre esse assunto advindos da Região Sul do país. Logo, quanto mais pesquisas houver envolvendo a temática de erros com medicação, mais subsídios para identificar as possíveis causas e buscar estratégias preventivas.

Pressupõe-se que o desenvolvimento desta pesquisa proporcionará um melhor conhecimento sobre o assunto, podendo, assim, implantar melhorias na instituição e na qualidade de seus serviços, focando funcionários e usuários, já que é uma instituição referência no Brasil, que apoia o ensino e a pesquisa e está em constante crescimento. Nesse ínterim, indaga-se: quais situações problemáticas permeiam a questão dos erros na administração de medicações realizada pelo técnico de enfermagem em uma Unidade de Terapia Intensiva (UTI) geral? Constituíram-se como objetivos: 1) descrever os tipos de erros ocorridos na administração de medicamentos pelos técnicos de enfermagem que trabalham em UTI geral; 2) identificar, na opinião dos técnicos de enfermagem, a(s) justificativa(s) para a ocorrência de erros de administração de medicamentos.

\section{METODOLOGIA}

Estudo qualitativo, exploratório, realizado na UTI adulta do Hospital Nossa Senhora da Conceição, em Porto Alegre/ RS, nos meses de janeiro de 2011 a fevereiro de 2011. Esse hospital oferece todas as especialidades de um hospital geral em seu ambulatório, na emergência e na internação, sendo que a emergência presta serviços 24 horas. Estimase que 31.800 pacientes são internados por ano na instituição, sendo que $54,5 \%$ são de Porto Alegre e $34 \%$ vêm da região metropolitana. A instituição disponibiliza um total de 801 leitos, e a UTI foi ampliada recentemente de 40 para 59 leitos, com o objetivo de qualificar ainda mais suas instalações, oferecendo um melhor atendimento a seus usuários, com o diferencial de boxes individualizados, equipamentos de ponta e uma central de monitoramento que pode acompanhar pacientes a distância. Essa estrutura a transformou em uma das maiores UTIs 100\% SUS do país. Os sujeitos da pesquisa são os técnicos de enfermagem, de todos os turnos, que tenham experiência profissional mínima de um ano na UTI, a ser estudada. Quanto ao número de participantes, obedeceu-se ao critério de saturação dos dados, totalizando 14 informantes. A entrevista semiestruturada ocorreu em um espaço reservado, dentro da própria instituição. Efetivaram-se três perguntas que permeiam aspectos relacionados à descrição dos tipos de erros ocorridos na administração de medicamentos pelos técnicos de enfermagem que trabalham em UTI geral e identificação, na opinião deles, da(s) justificativa(s) para a ocorrência de erros de administração de medicamentos. O projeto foi aprovado pelo Comitê de Ética e Pesquisa da instituiçãosede do estudo, com o número do protocolo projeto 10-209. Para resguardar o direito de confidencialidade dos sujeitos da pesquisa, as falas foram identificadas pela letra " $\mathrm{S}$ " mais um número atribuído a cada participante.

\section{APRESENTAÇÃO DOS RESULTADOS}

Dos 14 informantes, dez eram do sexo feminino. A idade

\begin{tabular}{|l|c|c|}
\hline \multicolumn{2}{|c|}{ TIPOS DE ERROS } \\
\hline ERROS & $\mathrm{N}^{\circ}$ & $\%$ \\
Preparação das medicações & 14 & 63,6 \\
Administração das medicações & 06 & 27,3 \\
Dispensação das medicações & 02 & 9,1 \\
\hline TOTAL & 22 & 100 \\
\hline
\end{tabular}

Fonte: Dados de pesquisa 
predominante foi de 20 a 30 anos, totalizando $50 \%$ dos entrevistados. Por sua vez, o tempo de experiência predominou de um a cinco anos (64,3\%) e de seis a dez anos (28,6\%).

A tabela 1 apresenta a distribuição de 22 erros de medicação cometidos pelos sujeitos da pesquisa, divididos em três categorias: erros na preparação das medicações, erros na administração das medicações e erros na dispensação das medicações.

A tabela 2 demonstra que as justificativas para ocorrências de erros com medicações estão divididas em quatro categorias: sobrecarga de trabalho, falta de atenção, inexperiência e problemas na estrutura.

\section{JUSTIFICATIVAS PARA OCORRÊNCIA DE ERROS}

\begin{tabular}{|l|c|c|}
\hline JUSTIFICATIVAS & $\mathrm{N}^{\circ}$ & $\%$ \\
\hline Sobrecarga de trabalho & 12 & 32,4 \\
Falta de atenção & 12 & 32,4 \\
Inexperiência & 07 & 19,0 \\
Problemas na estrutura & 06 & 16,2 \\
TOTAL & 37 & 100 \\
\hline
\end{tabular}

Fonte: Dados de pesquisa

\section{DISCUSSÃO DOS RESULTADOS}

\section{Erros de dispensação de medicamentos}

Em qualquer etapa do processo de terapia medicamentosa, podem ocorrer erros, desde a prescrição até a administração do medicamento, e esses erros representam de $65 \%$ a $87 \%$ dos eventos adversos. Tradicionalmente, os médicos prescrevem a terapia medicamentosa a ser administrada, e esse documento é referência para farmacêuticos e a equipe de enfermagem, para dar continuidade às etapas do processo(6).

A responsabilidade com os medicamentos é de vários profissionais da saúde: o médico, pela prescrição, a farmacêutica, pela dispensação e distribuição, e a equipe de enfermagem, pela administração e pelo monitoramento de possíveis reações adversas $^{(7)}$. Quanto à dispensação, os informantes sinalizam problemas com medicações que vêm trocadas e atrasadas:

"Hoje mesmo fui instalar um antibiótico que era Ampicilina + Sulbactan e quando fui pegar, a farmácia tinha dado Amoxacilina + Clavulanato, só que eu sempre leio muito, então, fui na farmácia e troquei" (S10).

"Nem sempre a medicação está pronta na farmácia, na hora que a gente tem que medicar; é certo, quase todo dia dá problema em relação a isso ali do turno da tarde" (S4).

Essa situação reforça a importância de cada etapa do processo de medicação, pois na etapa da preparação do medicamento foi identificado o erro. Caso contrário, o erro se consolidaria. O setor da farmácia nos hospitais, em especial, é um setor dinâmico, reflete o clima em que o hospital se encontra. Isso é, conforme as intercorrências nos diferentes setores, a farmácia reage atrasando e prejudicando a entrega dos medicamentos.

Um estudo realizado em um hospital público pediátrico no Espírito Santo apresentou como resultado uma porcentagem de $87,3 \%$ de erros de dispensação, relacionados aos erros de conteúdo. A taxa total de erros apresentada nesse estudo foi elevada comparada a estudos internacionais ${ }^{(8)}$.

\section{Erros de preparação de medicamentos}

É preciso consciência, segurança e conhecimento para efetuar a preparação de medicamentos. Dúvidas e dificuldades não esclarecidas levam à insegurança e à incerteza, e isso se torna um fator de risco para a ocorrência de erros no processo de preparação de medicamentos. Na fala a seguir, constatam-se erros em relação à diluição incorreta:

"Já presenciei alguns erros de colegas. Acho que todo erro é prejudicial, mas não foi tão grave, levando a óbito o paciente. Fazer uma medicação numa diluição não correta para a via correta, por ser uma via periférica, que requer uma diluição maior e muita paciência, se fizer uma diluição pura pode ocorrer uma flebite" (S3).

A diluição altera conforme a via de acesso. Medicações irritantes como cloreto de potássio e antibióticos podem ser administradas por via periférica, mas em maior tempo de infusão - em média quatro horas, para evitar, entre outros, o surgimento de flebites; se possui um cateter venoso central, o tempo de infusão pode ser menor, em torno de duas horas $^{(9)}$.

"Tem o potássio, que é um injetável; no cateter central, ele corre em duas horas, $e$ eu não me atentei, na verdade, são tempo e via, né. O paciente estava com cateter periférico, e infundi em duas horas. Não se queixou de dor porque os pacientes da UTI são sedados e entubados, então não teve nada, mas quando eu me dei conta foi assim, tipo, ocorreu num tempo, não chegou a dar flebite, mas pode dar, eu observei depois nos outros plantões" (S13).

O exemplo anterior foi agravado pelo fato de o funcionário não ter observado a via de acesso, conectando a medicação na dânula e iniciando a infusão. Como dito pelo informante, os pacientes da UTI, na maioria, encontram-se sedados e não referem dor ou qualquer outro desconforto.

Estudo realizado em uma instituição hospitalar universitária de São Paulo evidenciou que a maior porcentagem de dúvida de enfermeiros, técnicos e auxiliares de enfermagem foi quanto à questão de diluição dos medicamentos, totalizando 
$40,4 \%$. A relação da diluição do medicamento com a idade do paciente e com sua patologia existe, como em casos de pacientes com insuficiência renal e com distúrbios eletrolíticos ${ }^{(9)}$.

"Sei que já errei em relação à diluição; poderia ter diluído em mais e dilui em menos. Era uma polimixina, e isso difere de paciente para paciente. Pacientes renais geralmente usam diluição menor, fazer num volume menor, e não era paciente renal e a polimixina é uma medicação que é muito tóxica. Era 1 milhão duas doses de polimixina, mas nada aconteceu, só que poderia ter sido feito em mais" (S3).

A problemática continua, também, por não haver concordância entre a equipe médica e os farmacêuticos quanto às diluições.

"Acontecem esses erros muitas vezes por falta de comunicação do técnico com o enfermeiro ou com o próprio médico ou porque o médico quer que tu faça num determinado volume, mesmo que a farmacologia te diga que tu não pode. Tem muito isso aqui, de ter vários treinamentos com a farmácia que a gente aborda, tá, mais afinal é feito em qual volume, é feito em tanto, mas o médico pede que não seja, como já aconteceu. Aqui tem uma medicação que é hidrocortisona que, se tu fizer uma ampola de 50 miligramas, tu vai fazer em 100, se tu fizeres meia ampola tu vai diluir em 50 , tem médicos que querem que tu faça em 10, ai a gente até contesta, olha fazer em 20, então isso acontece muito também" (S3).

Apesar de ser o profissional mais qualificado, o farmacêutico, preparado para fornecer informações corretas, não é a fonte mais procurada para esclarecimentos. É preciso que tal profissional esteja presente nas instituições de saúde e seja envolvido em todas as etapas do processo, tendo nosso foco: o paciente ${ }^{(9)}$.

O erro de dose está intimamente ligado ao erro da leitura inadequada da prescrição ou leitura errada do tipo de medicação, como mostram as situações vivenciadas:

"Às vezes, é meio comprimido, e a gente escreve e faz um comprimido inteiro" (S5).

"Tinha uma dose de Cefepime que era pra fazer 1 grama de oito em oito horas. $\mathrm{Na}$ prescrição, a apresentação era Cefepime 2 gramas. A pessoa pegou e fez 2 gramas, eu vi porque a gente chega e tem que conferir tudo, teria que ter dos outros dois horários e só tinha um" (S11).

"Paciente que tinha a Fenitoína de $250 \mathrm{mg}$ tem $5 \mathrm{ml}$. Geralmente eles te pedem $100 \mathrm{mg}, 2 \mathrm{ml}$. Mas ela fez quatro ampolas, $1 \mathrm{~g}$, era $100 \mathrm{mg}$ e ela entendeu que era $1 \mathrm{~g}$ " (S11).

"Dose tipo Pipetazo é 4,5 g e tu não vê que está prescrito 2,25; é tão automático que tu faz 4,5" (S10).

"Já vi também acontecer da dose ser 500 mg e o colega não ler direito e achar que é $500 \mathrm{ml}$, fazendo uma superdosagem.
Foi bem complicado, ele fez toda a medicação, que veio para 24 horas, fez numa vez só" (S3).

"Paciente com taquicardia, foi solicitada a medicação Adenosina, que a gente só tem no carro de parada. A identificação da Adenosina estava numa divisória que de um lado ficava a Adenosina e, no outro, Deslanosídeo. Até não é uma coisa muito problemática, porque a função não é muito diferente, mas eu acabei pegando a medicação errada" (S1).

Um estudo ${ }^{(10)}$ constatou que $24,3 \%$ dos erros foram de dose, sendo que $50 \%$ foram relacionados à administração da medicação em dobro da dose prescrita, 27,7\% a metade da dose e $11,1 \%$ quatro vezes a dose prescrita. Por esse motivo, a dose unitária nas instituições é uma excelente estratégia na prevenção de erros.

\section{Erros de administração de medicamentos}

Um dos motivos apontados para a ocorrência de erros de administração de medicamentos foi a quantidade insuficiente da bomba de infusão, o que aumenta a probabilidade de erros no tempo de infusão dos medicamentos.

"A Vanco, a gente tem orientação de correr em uma hora, mas várias vezes acontece de correr em menor tempo. A Pipetazo também acaba correndo em menos tempo. Nem sempre tem bomba de infusão para controlar o tempo e, às vezes, acontece de correr rápido" (S4).

Outro problema frequentemente apontado são erros de via da administração.

"Uma colega fez uma medicação VO em um paciente entubado. Não tem como o paciente deglutir, então é uma coisa que pode ser bem séria. Graças a Deus nada aconteceu com o cliente" (S5).

"Já aconteceu uma vez de colocarem uma medicação enteral no acesso venoso" (S7).

"Já aconteceu de colocarem o álcool correr no lugar da água na sonda nasoentérica em bomba de infusão, que é uma das coisas que acho que aconteceram porque não leram e os frascos eram parecidos" (S13).

Dados apontados em um relatório de notificação da United States Pharmacopeia (USP) sinalizam que estudos apresentaram frequências de $18 \%$ e $19 \%$ de erros de via, entre todos os erros de medicação ${ }^{(6)}$. Para evitar erros de via, o profissional da saúde deve ler a prescrição médica com atenção e atentar para os cinco certos, que inclui via $\operatorname{certa}^{(10)}$.

A contínua educação dos profissionais da saúde pode auxiliar na diminuição desses erros com medicamentos. É indispensável a participação de todos os profissionais envolvidos nas etapas do processo de medicação, incluindo médicos, farmacêuticos, auxiliares de farmácia, enfermeiros, 
técnicos de enfermagem; todos, sem distinção, devem passar por reciclagens e treinamentos periódicos. Contudo, essas mudanças devem estar vinculadas a modificações de estrutura hospitalar, para serem eficazes.

\section{Justificativas para ocorrências de erros de medicações}

O contexto econômico e político atual interfere na regulação das relações no trabalho e, consequentemente, na política dos recursos humanos das organizações. Os gestores, na tentativa de diminuir seus problemas financeiros, optam por enxugar o número de funcionários, causando assim uma sobrecarga de trabalho aos que ficam ${ }^{(11)}$.

"Sobrecarga de trabalho, às vezes tu tá agitado e tem que fazer. Tá com dois pacientes, tá cuidando dos outros dois do teu colega, que os 15/20 minutos de intervalo tu tem que tá atento três, quatro pacientes, às vezes, então, pode acontecer, pede-me faz lá uma, sei lá, uma Amiodarona pro paciente do leito seis e fica com aquela do leito seis e faz no outro" (S2).

As instituições de saúde, em sua maioria, possuem um ritmo de trabalho bem acelerado, e as baixas remunerações obrigam os profissionais de saúde a terem mais de um emprego em diferentes turnos, para aumentar sua renda mensal, expondo o profissional a enormes riscos, como relata o sujeito a seguir:

"Muitas vezes trabalha em vários lugares, trabalha aqui, em outro, trabalha no turno da noite, no turno da manhã, cansado, a pessoa faz as coisas cansada, não lê direito e acaba errando, não adianta tu querer trabalhar 24 horas e chegar no último plantão, tu já tá muito cansado" (S3).

A excessiva carga de trabalho pode conduzir à insatisfação profissional e à exaustão, aumentando a rotatividade e o absenteísmo, comprometendo a imagem da instituição e o alcance de suas metas ${ }^{(12)}$.

"A ocorrência de erros é mais por excesso de trabalho. Isso aí faz com que cause uma desatenção, que daí tu não tem aquele tempo de ver e rever o que tu tá fazendo, que daí a gente passa correndo pelos 5/7 certos. Eu acho que um funcionário, principalmente da área da enfermagem, deveria ver e rever quando for aplicar, principalmente nesse caso de medicação. Ver de novo, identificar bem a medicação que tu tá fazendo" (S8).

Outro problema bem sinalizado foi a falta de atenção, como mostrado na tabela 2. Em estudo ${ }^{(4)}$ realizado, os profissionais apontaram como justificativa para os erros a falta de atenção e se culpavam, e não ao sistema em que estavam envolvidos, que pode ter grande parcela de culpa na ocorrência desses erros.

A pesquisa ora em análise apresentou um resultado um pouco diferenciado, já que os sujeitos da pesquisa associam a falta de atenção à sobrecarga de trabalho, como é demonstrado a seguir:

"Às vezes até por uma questão de agilidade ou de necessidade mesmo de preparar uma medicação na corrida e acaba incorrendo no risco de fazer um erro. Então, eu acho que ocorrência do erro é todo um contexto, falta de atenção com as condições que tu tem de desenvolver o trabalho" (S6).

A associação entre sobrecarga de trabalho e falta de atenção, somada à inexperiência, potencializa a ocorrência de erros. O número de escolas técnicas em nosso país aumentou significativamente nos últimos anos, com promessas de campos de trabalho, bons salários e baixas cargas horárias. Essa oferta exagerada de cursos, muitos com estrutura de docentes e de área física inadequadas, forma profissionais despreparados e desqualificados para enfrentar a complexidade que é uma unidade de saúde.

"Mas o Paracetamol, faço no tubo? Ela não sabia coisas básicas de medicação, acho que o pessoal é bem fraco, os que estão vindo. Ela perguntou se fazia EV ou pelo tubo; ela não sabia como é que ela fazia, não tinha a mínima noção. Às vezes parece que a pessoa vem leiga, não fez nem curso, coisas básicas. Acho que estão meio fracos os cursos" (S11).

Além disso, a UTI é setor complexo, que abrange pacientes em estado grave, que fazem uso de variados medicamentos potencialmente perigosos, com situação clínica instável. Sendo assim, erros de natureza medicamentosa agravariam ainda mais a situação do paciente, e o ato de medicar é uma das atividades mais praticadas dentro dessa unidade ${ }^{(3)}$. "Os novos acabam caindo dentro de uma UTI, que é um lugar que requer a tua atenção maior ainda que tu tem que ter nos outros lugares. Chegam aqui, pegam todo aquele processo que tem muito procedimento, dá muita intercorrência e não tem, não pegam bem essa parte de medicação e acabam fazendo as coisas na correria. Então eu acho que tem que haver um treinamento maior, um maior cuidado, maior atenção também do próprio técnico" (S3).

"A gente está com um número bem enxuto, então isso dá um excesso de trabalho para todos os profissionais ali, os técnicos de enfermagem, então isso facilita o erro? Acho que a pessoa erra quando está cheia de atribuições é muito mais fácil cometer o erro" (S7).

A UTI em estudo está em processo de ampliação de leitos e de número de profissionais da saúde. Vivenciam um momento em que ao mesmo tempo há insuficiência de profissionais e excessivo número de profissionais com pouco tempo de experiência na unidade. Estudos indicam que o insuficiente número de profissionais da enfermagem atinge negativamente o cuidado prestado aos pacientes, proporcionando maiores chances de ocorrência de erros adversos, entre eles os erros no processo da terapia medicamentosa $^{(12)}$. Logo, indaga-se como articular de modo positivo uma demanda aumentada de leitos de UTI pelo SUS e a exacerbada complexidade do tipo de pacientes que lá internam a um contingente profissional que necessita 
ser melhor instrumentalizado técnico e eticamente para o processo de cuidar em saúde.

\section{CONSIDERAÇÕES FINAIS}

Este estudo identificou e analisou erros de medicação e as respectivas justificativas para ocorrências desse tipo de erro, na opinião dos profissionais entrevistados em um hospital de grande porte em Porto Alegre/RS. Constatou-se que os erros na preparação dos medicamentos foram os mais frequentes, seguidos dos erros na administração e dos erros na dispensação. As justificativas sinalizadas foram a sobrecarga de trabalho e a falta de atenção, articuladas à inexperiência dos profissionais e aos problemas na estrutura.

A maior limitação deste estudo foi a dificuldade dos profissionais em expor seus erros e dialogar sobre o assunto. Ao falarem dos erros, reportavam-se a outros profissionais, e não às situações vivenciadas consigo mesmos. A aplicabilidade da punição tem sido considerada ineficaz na maioria das instituições de saúde; porém, inconsciente ou conscientemente, os profissionais continuam vivenciando esse medo, associado à vergonha. Quando ocorre o erro, a tendência é escondêlo, perdendo-se uma grande oportunidade de aprendizado. A mídia é um inimigo em potencial nessa questão, já que os profissionais se sentem expostos e desprotegidos. E a maior potencialidade deste estudo foi, também, a disponibilidade de a instituição aceitar a realização desse tipo de estudo. Estamos cientes de que essa problemática abrange várias outras instituições de saúde e espera-se que as mesmas aprimorem modos de identificar suas possíveis justificativas para a ocorrência de erros de medicamentos.

Conclui-se que a problemática do erro com medicamentos prejudica não apenas os sujeitos doentes, mas, também, os sujeitos que cuidam deles, nos aspectos profissional, pessoal, social e emocional. É premente a adoção de estratégias preventivas para evitar ou minimizar erros com medicamentos.

\section{Referências}

1. Vargas MAO, Luz AMH. Práticas seguras do/no cuidado de enfermagem no contexto hospitalar: é preciso pensar sobre isso e aquilo. Enferm Foco. 2010;1(1):23-7.

2. Santos JO. Sentimentos de profissionais de enfermagem após a ocorrência de erros de medicação. Acta Paul Enferm. 2007;20(4):483-8.

3. Toffoletto MC, Padilha KG. Consequências de medicação em unidades de terapia intensiva e semi-intensiva. Rev Esc Enferm USP. 2006;40(2):247-52. 4. Miasso Al. Erros de medicação: tipos, fatores causais e providências tomadas em quatro hospitais brasileiros. Rev Esc Enferm USP. 2006;40(4):524-32. 5. Carvalho VT, Cassiani SHB. Erros na medicação e consequências para profissionais de enfermagem e clientes: um estudo exploratório.

Rev Latinoam Enferm. 2002;10(4):523-9.

6. Gimenes FRE. Administração de medicamentos, em vias diferentes das

prescritas, relacionada à prescrição médica [Internet, citado em 2011 mai 08].

Disponível em: http://www.scielo.br/pdf/rlae/v19n1/pt_03.pdf.

7. Cassiani SHB. O sistema de medicação nos hospitais e sua avaliação por um grupo de profissionais. Rev Esc Enferm USP. 2005;39(3):280-7.

8. Costa LA, Valli C, Alvarenga AP. Erros de dispensaçăo de medicamentos em

um hospital público pediátrico [Internet, citado em 2011 mai 05]. Disponível em:

http://www.scielo.br/pdf/rlae/v16n5/pt_03.pdf.

9. Silva DO. Preparo e administração de medicamentos: análise de

questionamentos e informaçōes da equipe de enfermagem [Internet,

citado em 2011 Mai 08]. Disponivel em: http://www.scielo.br/pdf/rlae/

v15n5/pt_v15n5a19.pdf.

10. Teixeira TCA, Cassiani ISHB. Análise de causa raiz: avaliação de erros de

medicação em um hospital universitário. Rev Esc Enferm USP. 2010;44(1):139-46.

11. Freitas GF, Fugulin FMT, Fernandes MFP. A regulação das relaçōes de trabalho e o gerenciamento de recursos humanos em enfermagem. Rev Esc Enferm USP. 2006;40(3):434-8.

12. Cucolo DF, Perroca MG. Reestruturação do quadro de pessoal de enfermagem e seu impacto sobre as horas de assistência. Rev Latinoam Enferm. 2010;18(2):31-9. 